\title{
THE DEPRIORITISED APPROACH TO PRIORITISED ALGORITHMS
}

\author{
STEPHEN HOWE
}

(Received 18 February 2009)

2000 Mathematics subject classification: primary 05C80, 68W20; secondary 05C20, 05C69.

Keywords and phrases: randomised algorithms, deprioritised algorithms, random regular digraphs, dominating sets.

Randomised algorithms are an effective method of attacking computationally intractable problems. A simple and fast randomised algorithm may produce results to an accuracy sufficient for many purposes, especially in the average case. In this thesis we consider average case analyses of heuristics for certain NP-hard graph optimisation problems. In particular, we consider algorithms that find dominating sets of random regular directed graphs. As well as providing an average case analysis, our results also determine new upper bounds on domination numbers of random regular directed graphs.

The algorithms for random regular directed graphs considered in this thesis are known as prioritised algorithms. Each prioritised algorithm determines a discrete random process. This discrete process may be continuously approximated using differential equations [4]. Under certain conditions, the solutions to these differential equations describe the behaviour of the prioritised algorithm. Applying such an analysis to prioritised algorithms directly is difficult. However, we are able to use prioritised algorithms to define new algorithms, called deprioritised algorithms, that can be analysed in this fashion.

Defining a deprioritised algorithm based on a given prioritised algorithm, and then analysing the deprioritised algorithm, is called the deprioritised approach. The initial theory describing the deprioritised approach was developed by Wormald [5] and has been successfully applied in many cases [1-3]. However, not all algorithms are covered by Wormald's theory: for example, algorithms for random regular directed graphs. The main contribution of this thesis is the extension of the deprioritised

Thesis submitted to The University of New South Wales, September 2008. Degree approved, January 2008. Supervisor: Dr Catherine Greenhill.

(C) 2009 Australian Mathematical Society 0004-9727/2009 \$16.00 
approach to a larger class of prioritised algorithms. We demonstrate the new theory by applying it to two algorithms which find dominating sets of random regular directed graphs.

\section{References}

[1] M. Beis, W. Duckworth and M. Zito, 'Packing vertices and edges in random regular graphs', Random Structures Algorithms 32 (2008), 20-37.

[2] J. Diaz, M. Serna and N. C. Wormald, 'Bounds on the bisection width for random $d$-regular graphs', Theoret. Comput. Sci. 382 (2007), 120-130.

[3] W. Duckworth and N. C. Wormald, 'On the independent domination number of random regular graphs', Combin. Probab. Comput. 15 (2006), 513-522.

[4] N. C. Wormald, 'The differential equation method for random graph processes and greedy algorithms', in: Lectures on Approximation and Randomized Algorithms (Polish Scientific Publishers PWN, Warsaw, 1999), pp. 73-155.

[5] _ 'Analysis of greedy algorithms on graphs with bounded degrees', Discrete Math. 273 (2003), 235-260.

STEPHEN HOWE, Centre for Mathematics and its Applications, Mathematical Sciences Institute, Australian National University, Canberra, ACT 0200, Australia e-mail: stephen.howe@anu.edu.au 